\title{
Time to Guide: Evidence for Delayed Attentional Guidance in Contextual Cueing
}

\author{
Melina A. Kunar ${ }^{1}$, Stephen J. Flusberg ${ }^{2}$, and Jeremy M. Wolfe 3,4 \\ Department of Psychology, The University of Warwick, Coventry, CV4 7AL, UK
}

\begin{abstract}
Contextual cueing experiments show that, when displays are repeated, reaction times (RTs) to find a target decrease over time even when the observers are not aware of the repetition. Recent evidence suggests that this benefit in standard contextual cueing tasks is not likely to be due to an improvement in attentional guidance (Kunar, Flusberg, Horowitz \& Wolfe, 2007). Nevertheless, we ask whether guidance can help participants find the target in a repeated display, if they are given sufficient time to encode the display. In Experiment 1 we increased the display complexity so that it took participants longer to find the target. Here we found a larger effect of guidance than in a condition with shorter RTs. Experiment 2 gave participants prior exposure to the display context. The data again showed that with more time participants could implement guidance to help find the target, provided that there was something in the search stimuli locations to guide attention to. The data suggest that although the benefit in a standard contextual cueing task is unlikely to be a result of guidance, guidance can play a role if it is given time to develop.
\end{abstract}

\section{Introduction}

Locating a desired target item in a scene with a number of competing distractors is termed visual search. Visual search is an activity that we usually perform many times each day. Common examples might include looking for your car in a car park or for a particular student in a lecture theatre. In contrast to many laboratory search tasks, in these real world situations, the target is embedded in a surrounding context.

Recent research has explored the effects of context on visual search. Context is known to speed object detection (Biederman, 1972). For example, we are faster at naming a traffic light if it appears in a street scene rather than in a kitchen scene. Research by Chun and Jiang (1998; 2003) confirms this intuitive prediction, even in the absence of semantic cues. Using a simple search task where participants were asked to find a letter T among letter Ls, for example, they demonstrated that the spatial layout or context of a display could influence how quickly participants found a target. In a series of studies they found that if the target item was embedded in an invariant configuration that was repeated across the experiment, reaction times (RTs) to find the target were quicker than when the target appeared in a novel or unrepeated configuration. This RT benefit is termed "contextual cueing". Further research found that contextual cueing relies on implicit memory, is learned after only 5 repetitions of the display (Chun \& Jiang, 1998), and can persist for up to a week (Chun \& Jiang, 2003).

\footnotetext{
E-mail: M.A.Kunar@warwick.ac.uk Tel: +44 (0)2476 522133 Fax: +44 (0)2476 524225

${ }^{1}$ University of Warwick

2 Stanford University

${ }^{3}$ Harvard Medical School

${ }^{4}$ Brigham \& Women's Hospital
} 
In their initial paper, Chun and Jiang (1998) suggested that contextual cueing occurs because the visual context can guide spatial attention towards the target. In fact, it has been assumed by most of the literature, that contextual cueing arises due to a guidance benefit (e.g., Chun, 2000; Chun \& Jiang, 1998; Chun \& Jiang, 1999; Chun \& Jiang, 2003: Endo \& Takeda, 2004; Hoffmann \& Sebald, 2005; Jiang \& Chun, 2001; Jiang \& Leung, 2005; Jiang, Song \& Rigas, 2005; Jiang \& Wagner, 2004; Lleras \& Von Mühlenen, 2004; Olsen \& Chun, 2002; Tseng \& Li, 2004). Recently, we tested this assumption and found that this prior belief was, for the most part, erroneous (Kunar, Flusberg, Horowitz \& Wolfe, 2007). In visual search we can measure the benefit of guidance or search efficiency by plotting the RT $\times$ set size (i.e. number of distractors plus target) function and derive the best-fitting linear equation. The slope of this line expresses the efficiency of search (in msec/item), while non-search factors (such as perceptual or response selection factors) are relegated to the intercept. Guidance, by definition, has its effect on search slopes as it effectively reduces set size by guiding attention to some items in preference to others. If one is searching for a red " $\mathrm{T}$ " among Ls evenly divided between red and green, attention will be guided to the red items. This will cut the relevant set size in half and cut the RT $\times$ set size slope to half what would be found for the same search without color guidance (Egeth, Virzi \& Garbart, 1984).

Using this logic, Kunar et al. (2007) argued that if contextual cueing occurred due to an improvement in guidance, search slopes should be more efficient when the context is repeated than when it is unrepeated. However, the data show that there was no statistical difference between slopes in repeated and unrepeated conditions (see Kunar, Flusberg \& Wolfe, 2006 and Kunar, Flusberg, Horowitz \& Wolfe, 2007). Furthermore, we found other evidence suggesting that contextual cueing did not behave like attentional guidance. Firstly, we know that we can guide our attention to multiple locations within a display. For example, the color red can be used to guide attention to many possible target locations. In contrast, however, we found that a repeated context could only provide a benefit for, at the very best, one or two target locations (Kunar, Michod \& Wolfe, 2005). Secondly, with standard guidance it makes very little difference if a target on one trial appeared in a location occupied by a distractor on the previous trial. However, this was not the case in contextual cueing: If a distractor occupied a previous target location then no contextual cueing effect was found (Kunar et al., 2005).

Thirdly, we found a small contextual cueing effect occurred even when the target "popped out". That is, contextual cueing occurs even when the efficiency of search was already maximal (Kunar et al., 2007). From this, we hypothesize that contextual cueing may be, at least in part, due to response selection processes. That is, if a participant has seen a display repeatedly they may require less evidence (and thus less time) before being able to commit to a response. To use a real-life analogy it may be faster to respond to a student if seen in the familiar and repeated context of a lecture theatre than if seen in a novel surrounding, such as a shopping mall (please note that the actual response across repeated situations may vary, however it is your 'willingness' to commit to a response in a repeated context that is facilitated).

It appears unlikely that attentional guidance is the primary mechanism of the standard contextual cueing effect. Still it is worth asking if guidance can ever play a role? Given an invariant context, which always predicts the target to be in location XY, it seems counterintuitive that we cannot use this to guide our attention. Imagine walking into a familiar office to make a phone call. In this case, you would not need to search the desk to find the phone. If you were familiar with the layout you should just move your attention straight to the object in question (in fact, Brockmole \& Henderson, 2006a, showed evidence supporting this where fewer eye movements were made when searching for a target in a repeated real scene photograph compared to a novel scene). What makes searching a familiar context such as the office and searching a repeated display for a target different? There are many possible answers, but one difference, in particular, is the time taken to respond to the target. In laboratory visual 
search tasks, response times are usually in the milliseconds, however, response times to items in an office may take several seconds. Given this difference, we ask whether lengthening the time prior to response, in a contextual cueing task, would allow you to use attentional guidance? In other words, in complex displays, does context take time to guide?

In Experiment 1, we increased the complexity of the background, in a contextual cueing paradigm, so that response times took longer. This was done by overlaying the search stimuli onto a background grid, containing lines made up to resemble a brick wall. Pilot work has shown that these displays generally increase RTs (see also Wolfe et al., 2002). If, giving more time to process the display allows participants to implement guidance, we would expect to find a bigger reduction in search slopes in the complex display compared to a display with no background. The data showed that this was the case. Experiment 2 again gave participants more time with the display context, but this time without increasing the display complexity. The overall context of the display was marked out by placeholders and masks that offset after a delay to produce the search stimuli. The end display to be searched was identical to a standard contextual cueing baseline condition. However, the data showed that again with more time, search slopes in contextual cueing tasks were reduced. This guidance benefit was only fully observed, however, when there were items in the exact locations where the search stimuli would appear. When the context was marked out just by empty placeholders, there was little improvement in guidance. Taken as a whole the results suggest that guidance can occur within contextual cueing, as long as there is adequate time to parse the display.

\section{Experiment 1}

\section{Method}

Participants-Twelve individuals between the ages of 18 and 55 years served as participants. Each participant passed the Ishihara test for color blindness and had normal or corrected to normal vision. All participants gave informed consent and were paid for their time.

Stimuli-The experiment was conducted on a Macintosh computer using MatLab Software with the PsychToolbox (Brainard, 1997; Pelli, 1997). The distractor items were L shapes presented randomly in one of four orientations $(0,90,180$ or 270 degrees). The target item was a $\mathrm{T}$ shape rotated 90 degrees either to the left or to the right with equal probability. There was always a single target present. A blue dot at the center of the screen served as a fixation point. The background of the screen was dark grey, and at the center was a white grid, outlined in black, subtending a visual angle of $29.5^{\circ} \times 29.5^{\circ}$. Stimuli appeared on three imaginary concentric circles surrounding the fixation point with diameters of $9.5^{\circ}, 15.5^{\circ}$, and $25^{\circ}$.

Stimuli could appear randomly in one of sixteen evenly spaced locations on each imaginary circle. Both the Ls and the Ts were made up of two black lines of equal length. Because visual acuity declines as function of the distance from the fixation point, the size of the lines increased the further they were from the focal point on the screen. The lines on the smallest imaginary circle subtended $1^{\circ}$ in length, those on the middle imaginary circle subtended $1.5^{\circ}$, and those on the largest imaginary circle subtended $2.5^{\circ}$.

In the standard contextual cueing conditions, the background of the display was simply white, while in the complex-background condition, the background included a "wall" composed of black lines forming squares subtending $5.9^{\circ} \times 5.9^{\circ}$ visual angle. There were five rows of squares, and each row was slightly offset, resembling the formation of a brick wall. Specifically, the top row, middle row, and bottom row consisted of five full squares, while the two intervening rows consisted of four full squares and two half-squares on either end. At the center of each of the left and right sides of the squares was a hole subtending a visual angle of $1.5^{\circ}$. 
There were two conditions in this experiment: a 'Standard' contextual cueing task and a 'Wall' contextual cueing task. For the Standard contextual cueing task, there were two blocks, one with a set size of 4 and one with a set size of 12. For the Wall condition, there were four blocks, with set sizes of 1, 4, 8 and 12 respectively. For the set size 1 display, the context was marked out by twelve circular placeholders. The target, $\mathrm{T}$, appeared within one of these placeholders while the rest of the placeholders remained empty. Half the circular placeholder contexts were predictive of the target location, whilst the remaining contexts were randomly generated (see also Kunar, Flusberg, Horowitz \& Wolfe, 2007). The set size 1 display was used to determine whether, even with 'perfect' guidance, a contextual cueing effect was still observed (it is unlikely that participants were searching the background here as a wall background has been previously shown to only affect the intercept and not affect the search process, Wolfe et al., 2002), whereas the set size 8 display was simply to confirm that an RT contextual cueing effect was observed with the wall background. RTs from set size 4 and 12, in both the Standard and Wall contextual cueing tasks were used to generate search slopes (as well as being used to show an RT contextual cueing effect).

In each block, there were 7 epochs each of 64 trials each. Within each epoch, half the trials had fixed configurations that were repeated throughout the experiment (predictive displays). These consisted of 4 fixed displays that were repeated 8 times within an epoch. Thus, over 7 epochs, each repeated display was shown 56 times. The other half of the trials had novel configurations on each trial (random displays). If just 4 target locations were repeated on half the trials (predictive displays) while target locations were random on the half (the random displays), participants might learn that the 4 repeated locations were favored regardless of context. To prevent this, only four target locations were used for the random displays (see also Chun \& Jiang, 1998). In these random displays the target locations were never correlated with the pattern of distractor locations. Thus, in the predictive displays, each of 4 target locations was associated with a specific context while in the random displays, each of different 4 target locations was presented on a random set of non-predictive contexts. Each display was visible until response. If no response had been recorded within 10 seconds the display disappeared and the next trial was initialized.

Procedure-Participants were asked to respond to the direction of the target letter $\mathrm{T}$ by pressing the letter ' $a$ ' if the stem of the T was pointing left and the quote (") key if the stem of the $\mathrm{T}$ was pointing right. Please note that both here and in Experiment 2 the target could point either left or right on any trial and thus, from trial to trial, could vary its orientation within a repeated display. A tone sounded at the start of each trial, at which point the items appeared on the screen. Participants were asked to respond as quickly but as accurately as possible and feedback was given after each trial. Participants completed all six blocks and the order of blocks was randomized. Each condition consisted of 10 practice trials and 448 experimental trials. Example displays can be found in Figure 1.

Data analysis-In the literature, there have been many ways to formally define contextual cueing. Chun \& Jiang (1998) suggested that the contextual cueing effect should be measured as the difference between predictive and random configurations across the last three epochs (see also Jiang, Leung \& Burks, submitted, Kunar, Flusberg \& Wolfe, 2006, and Kunar, Flusberg, Horowitz \& Wolfe, 2007). This procedure focuses on the asymptotic benefit for having learned a predictive context over a random one. Following their reasoning, we collapsed the data across the last 3 epochs (here epochs 5 to 7 ) and used this as the standard measure of contextual cueing. 


\section{Results and Discussion}

Overall error rates were quite low at $4 \%$. There was a significant main effect of configuration on errors, $\mathrm{F}(1,11)=5.6, \mathrm{p}<0.05$, and of set size, $\mathrm{F}(1,11)=5.1, \mathrm{p}<0.05$; random trials showed a higher error rate than predictive and there were more errors at set size 4 than at set size 12 . As there was nothing of interest in the error rates we do not report them further. RTs below $200 \mathrm{msec}$ and above $4000 \mathrm{msec}$ were removed. This led to the removal of less than $1 \%$ of the data. Please note that there were a number of analyses that could be run for this experiment. However, to be concise we only report the statistics relevant to the question in hand. Figure 2 shows RTs for both predictive and random configurations for all set sizes.

As expected, an RT contextual cueing effect was observed for all set sizes within all conditions. Table 1 shows the asymptotic difference between predictive and random RTs across the last 3 epochs. Both the Standard and Wall contextual cueing tasks showed that RTs to predictive displays were faster than those to random displays. Corresponding data was again found when we examined the main effects from each condition: in all conditions, RTs were faster in predictive contexts compared to random (all Fs $>5$, ps $<0.05$, except set size 4 of the Wall condition, which showed a marginal effect, $\mathrm{F}(1,11)=4.3, \mathrm{p}=0.06)$. The data showed that a contextual cueing effect could be found even when the complexity of the background was increased and also when the target was presented in isolation (this latter effect replicates our previous findings, where contextual cueing was shown in situations where guidance was already 'perfect', Kunar et al., 2007). The repeated contexts were learned quickly. From Figure 2 it seems that, in most conditions, a contextual cueing effect is already observed by the end of Epoch 1. This is not surprising if we remember that by this point participants had seen each repeated display eight times. In the standard contextual cueing experiments an RT advantage is normally apparent after at least 5 repetitions of the predictive display (e.g. Chun \& Jiang, 1998). To examine the time course of contextual cueing in our Experiments Figures $3 \mathrm{a}$ and $3 \mathrm{~b}$ show RTs for the first four repeats of the predictive and random displays in the Wall and Standard contextual cueing conditions, respectively (set sizes 4 and 12). It is clear that at the beginning of the experiment (i.e., on their first exposure) RTs for the predictive trials do not differ from their random counterparts (all p's $>0.6$ ). However, as expected, with increased repetitions RTs from predictive displays become faster than those from repeated displays.

The central issue for present purposes involves the search slopes: Did the slopes differ as a function of the time taken to find the target? Overall, RTs were longer in the Wall conditions compared to the Standard contextual cueing conditions, $\mathrm{F}(1,11)=24.4, \mathrm{p}<0.01$. Did this increased exposure produce a bigger benefit in attentional guidance? We suggest that it did. Figure 4 shows search slopes across epochs, whereas Table 2 shows the asymptotic difference between predictive and random search slopes over the last 3 epochs and the difference in predictive and random slopes at Epochs 1 and 7.

There is a statistically weak improvement in efficiency even in the Standard conditions of this experiment. Search in predictive trials was marginally more efficient than that in random trials (comparing the last three epochs: $\mathrm{t}(11)=1.9, \mathrm{p}=0.08$; comparing all epochs: $\mathrm{F}(1,11)=5.1$, $\mathrm{p}<0.05)$. These results are different than our previous failure to find a slope difference in contextual cueing. We shall discuss this discrepancy momentarily. First, however, let us examine data from the Wall condition. Again these results showed that search slopes for predictive displays were more efficient than those from random trials. This was true both in the asymptotic difference over the last 3 epochs, $\mathrm{t}(11)=3.3, \mathrm{p}<0.01$ and in the main effect across all trials, $\mathrm{F}(1,11)=8.6, \mathrm{p}=0.01$. The improvement in search efficiency was greater when there was more time to process the display. Examining the interaction between the condition (i.e., Wall vs. Standard) and configuration (i.e. predictive vs. random), we see that there was a bigger difference between random and predictive slopes in the Wall condition compared to the Standard contextual cueing condition, $F(1,11)=7.5$, $p<0.05$ (Table 2). Search 
efficiency improved by approximately $16 \mathrm{msec} / \mathrm{item}$ in the wall case, compared to $7 \mathrm{msec} / \mathrm{item}$ in the standard case. When it took longer to respond to the target, there was a larger effect of guidance. Furthermore, Table 2 shows that the effect of guidance behaves differently in the Standard and Wall conditions. Although there was an initial benefit in search slope at Epoch 1 for the Standard condition, this effect had decreased by Epoch 7. In contrast, we find that the guidance benefit increased between Epoch 1 and Epoch 7 in the Wall condition. This latter result would be expected if participants were learning to guide to the target location when given increased exposure to the display.

Why is there a slope benefit in the Standard contextual cueing condition in this experiment? As mentioned above, these data contradict our previous findings (across fifteen separate experiments) where there was little benefit in search slopes in standard contextual cueing tasks (see Kunar, Flusberg \& Wolfe, 2006 and Kunar, Flusberg, Horowitz \& Wolfe, 2007, for details). One possibility may be that there is a limited amount of guidance in contextual cueing. Chun and Jiang (1998) reported a small reduction in search slopes in predictive displays compared to random. It may be that this weak benefit in guidance was reflected in the search data here. However, this seems an unsatisfactory answer given that such a benefit has not been found in a large number of other experiments (Kunar, Flusberg \& Wolfe, 2006; Kunar, Flusberg, Horowitz \& Wolfe, 2007, see also Experiment 2 here). An alternative explanation is that participants were able to transfer skills learned in the Wall conditions to the Standard condition, skewing the data to show a guidance effect. In fact, pilot data suggests that this is quite possible. Here, when participants were trained to use guidance in one task prior to completing a standard contextual cueing task then a guidance benefit was observed. The guidance benefit was not seen when no guidance training was given. However, further experimentation needs to be conducted before we can conclude anything more concrete.

The important point from this experiment was that there was a larger effect of guidance when it took longer to respond to the target. One could argue, however, that it was the complexity of the background in the Wall condition rather than the longer search time that led to the larger guidance effect. To examine this, we kept the background complexity constant in Experiment 2 , but varied the time available to process the context prior to the appearance of the search stimuli. In this experiment and a number of replications, we again observed a larger guidance effect when there was more time to process the display context.

\section{Experiment 2}

\section{Method}

Participants-Thirteen individuals between the ages of 18 and 55 years served as participants. Each participant passed the Ishihara test for color blindness and had normal or corrected to normal vision. All participants gave informed consent and were paid for their time.

Apparatus and Stimuli-The apparatus was similar to Experiment 1. The stimuli were changed slightly. A blue dot at the center of the screen served as the fixation point. The background of the display was dark grey. As cartooned in Figure 5, three black concentric circles surrounded the focal point with diameters of $9.5^{\circ}, 15.5^{\circ}$, and $25^{\circ}$. Sixteen black lines radiated out from the focal point roughly equidistant from one another to form a webbed lattice. The stimuli were located in placeholder circles that appeared at the intersections between the concentric circles and the spokes. The outline of the circles was white, the inside of the circles was the color of the background, and the stimuli were white. Because visual acuity declines as function of the distance from the fixation point, the size of the placeholders increased the further they were from the fixation point on the screen. Those on the closest concentric circle subtended $2^{\circ}$ in diameter, those on the middle concentric circle subtended $3.3^{\circ}$, and those on the furthest concentric circle subtended 5.4. 
The stimuli consisted of white lines in various orientations. There were four distractor lines angled $30^{\circ}$ and $60^{\circ}$ to the left and right of the vertical. The target was either a vertical or horizontal line. Stimuli appearing in the smallest placeholders subtended a visual angle of $1.5^{\circ}$, those in the middle placeholder subtended $2.3^{\circ}$, and those in the largest placeholders subtended $3.5^{\circ}$.

There were three conditions: a "Standard" contextual cueing task; an "SOA onset" condition where the stimuli appeared $800 \mathrm{msec}$ after the placeholders onset and an "SOA Offset" condition where a mask initially appeared within the placeholders. The offset of the mask revealed the stimuli after an $800 \mathrm{msec}$ SOA. The masks consisted of six angled white lines (vertical, horizontal, and four lines rotated $30^{\circ}$ and $60^{\circ}$ on both sides of vertical respectively), which overlapped each other to form an asterisk. The offset of a mask would leave one line present as part of the search array. Two blocks of each condition were run: one with a set size of 8 and the other with a set size of 12 .

As in Experiment 1, there were 7 epochs per block, each with 64 trials in each epoch. Half the overall trials had fixed configurations that were repeated throughout the experiment (predictive displays). These consisted of 4 fixed displays that were repeated 8 times within an epoch. Each repeated display was shown 56 times throughout the experiment. The other half of the trials had a novel configuration that was generated at random. In order to ensure that participants were not simply learning absolute target locations from the predictive displays, targets in the random displays appeared equally often in just 4 randomly selected locations. However, these appearances were not correlated with any pattern of distractor locations. The displays were visible until response. If no response had been recorded within 10 seconds the next trial was initiated.

Procedure-Participants were asked to look for a vertical or horizontal line among oblique lines. They pressed the letter "a" if the line was vertical and the quote key (") if the line was horizontal. A tone sounded at the start of each trial, at which time the placeholders appeared on the screen. In the standard contextual cueing condition the stimuli appeared concurrently with the placeholders. In the SOA Onset condition, the target and distractor stimuli appeared $800 \mathrm{msec}$ after the onset of the placeholders. In the SOA Offset condition the placeholders and the masks appeared concurrently. The masks then offset to produce the target and distractor stimuli $800 \mathrm{msec}$ later. Participants were asked to respond as quickly but as accurately as possible to the orientation of the target and feedback was given after each trial. Participants completed all six blocks and the order of blocks was randomized. Each condition consisted of 10 practice trials and 448 experimental trials.

\section{Results and Discussion}

The data from one participant was discarded due to high error rates (36\%). For the remaining twelve participants, overall error rates were low at $3.5 \%$. None of the main effects or interactions of errors were significant and as there was nothing of interest in the error rates we do not report them further. RTs below $200 \mathrm{msec}$ and above $4000 \mathrm{msec}$ were removed. This led to the removal of $3 \%$ of the data. Figure 6 shows RTs for both predictive and random configurations for each condition.

Examining the RTs, a standard contextual cueing effect was found for every condition at every set size. In each condition, RTs in predictive displays were faster than those in random displays (all Fs $>13$, ps $<0.01$ and all Fs $>6$, ps $<0.05$, for set size 8 and 12 respectively). Table 1 shows that this difference was also reliable when we compared performance over the last 3 epochs. Again in each condition predictive RTs were faster than random. 
As in Experiment 1, it is the search slopes that are critical for present purposes. Figure 7 shows the effect of search slope over epoch for each condition and Table 3 shows the difference in predictive and random search slopes over the last 3 epochs. Crucially, a search slope benefit was only observed for the SOA Offset condition. In that condition, there was an asymptotic difference over the last 3 epochs where search slopes from predictive displays were more efficient than those from random, $t(11)=2.9, p=0.01$. Furthermore, there was a marginal effect across all epochs, $\mathrm{F}(1,11)=3.2, \mathrm{p}=0.1$. We have replicated this effect several times (see below).

In contrast, as can be seen from Figures 7 and Table 3, the Standard condition produced no guidance benefit of a predictive context over a random one $(\mathrm{t}(11)=0.3, \mathrm{p}=\mathrm{n} . \mathrm{s}$. and $\mathrm{F}(1,11)$ $=0.0, p=$ n.s., for the asymptotic effect and overall main effect respectively). This replicates the data from Kunar et al. (2007). In the SOA Onset condition, at first glance, the data suggests that there might be a difference in search slope. However, these results do not stand statistically. Overall, there was no asymptotic difference in the last 3 epochs, $t(11)=1.1, \mathrm{p}=\mathrm{n}$.s., nor a main effect between predictive and random search slopes, $\mathrm{F}(1,11)=0.6, \mathrm{p}=\mathrm{n}$.s.. Although there was a numerical trend suggesting that placeholders themselves may improve search efficiency, it seems that for the strongest benefit in guidance to occur, there need to be actual stimuli in the soon-to-be searched stimuli locations. In other words, there needs to be something to guide to. As a search slope effect occurred in the SOA Offset condition using the same number of participants as in the SOA Onset condition, it seems unlikely that the lack of a slope effect in the SOA Onset condition is due to a lack in power. Again other studies support this conclusion (see below).

Overall, the data show that there was a contextual cueing benefit in search efficiency when participants were given additional time to process the display context and when placeholders occupied locations of eventual search. We have replicated this effect in two other studies; one where SOA Onset conditions of 400 and $800 \mathrm{msec}$ were used and one where SOA Offset conditions of 400, 800 and $1200 \mathrm{msec}$ were used. The slope differences between predictive and random displays are shown in Figure 8. Again the results replicate the findings above: none of the SOA Onset conditions showed a reliable slope difference $(\mathrm{t}(15)=0.8, \mathrm{p}=\mathrm{n}$.s., and $\mathrm{t}(15)=1.4, \mathrm{p}=\mathrm{n}$.s., for SOAs 400 and $800 \mathrm{msec}$ respectively), whereas all of the SOA Offset conditions did $(\mathrm{t}(12)=2.3, \mathrm{p}<0.05 ; \mathrm{t}(12)=2.4, \mathrm{p}<0.05$ and $\mathrm{t}(12)=3.0, \mathrm{p}=0.01$ for SOAs 400,800 and $1200 \mathrm{msec}$ respectively). The data add complementary evidence suggesting that a guidance benefit occurs if participants were given prior exposure to the display context. This benefit was not observed, however, if there was nothing in the exact locations of where the search stimuli would appear. We discuss this further in the General Discussion.

\section{General Discussion}

Contextual cueing shows that repeating a display over time reduces RTs to find the target compared to displays that are not repeated. We have previously shown that this benefit in RT is not likely to be due to an improvement in attentional guidance in the standard contextual cueing conditions. Our evidence for this is that search slopes do not become more efficient in contextually cued displays (Kunar et al. 2007, see also Experiment 2 in this paper). Here we have shown that context can guide attention but it takes a while to manifest. Experiment 1 used a complex background display (the Wall contextual cueing condition) to slow search relative to a Standard contextual cueing condition with a blank background. The addition of the wall to the display increased search complexity, thereby increasing the time taken to find the target (see also Wolfe et al., 2002). With this extra time to process the display, we found a greater guidance benefit, in terms of improved search efficiency, compared to the Standard contextual cueing case. Experiment 2 again showed that previewing the context improved search efficiency but only when there was something to guide to (i.e. when there were masks present 
in the SOA Offset condition). The data suggest that guidance can play a role in contextual cueing as long as there is sufficient time to parse the display. However in a standard contextual cueing experiment the RT benefit does not seem to be driven by an improvement in attentional guidance (Kunar et al., 2007).

Peterson and Kramer (2001) investigated eye movements in contextual cueing. With a single set size, they could not calculate search slopes. Nevertheless, they found that contextual cueing did not provide 'perfect guidance' on every trial. However, it did increase the proportion of trials on which the gaze went to the target on the first saccade and it did reduce the number of saccades necessary to find the target in repeated conditions. Peterson \& Kramer (2001) suggested that recognition of the display was not always perfect, but that when the display was recognized, attention (in their case, as indicated by eye movements) moved directly to the target. Furthermore, recognition of the display did not occur on some trials until later on in the search process. This idea falls in line with our data. Guidance to a familiar display does not occur immediately, and can only be implemented over time - perhaps, in accordance with Peterson and Kramer's work, once the display has been recognized.

In fact, it is possible that explicit recognition may play a role in whether guidance is used in search or not. Evidence for this possibility can be derived from studies where participants were contextually cued by the background properties of the display (Kunar, Flusberg \& Wolfe, 2006). As in contextual cueing from spatial layout, there was an RT benefit of having a predictive background but no benefit to search slope. However, if participants were explicitly alerted, and given $1500 \mathrm{msec}$ to process the background prior to the onset of the stimuli, then search slopes were reduced to 0 msec/item in $64 \%$ of the participants (and were significantly reduced in the other $36 \%$ ). Given time and explicit awareness of a display's predictability enabled participants to implement attentional guidance.

A similar finding was reported by Brockmole and colleagues (e.g., Brockmole \& Henderson, 2006b; Brockmole, Castelhano \& Hendeson, 2006) who investigated contextual cueing using real scenes. As mentioned in the introduction, Brockmole and Henderson (2006a) found that fewer eye movements were made when the target was in a repeated real scene compared to when it was in a novel scene. This suggests that participants were utilizing aspects of the display to guide their attention to. However unlike the implicit learning in standard contextual cueing tasks (e.g. searching for a T among Ls, Chun \& Jiang, 1998 and Experiment 1 here), memory for real scene-target covariation was shown to be explicit (Brockmole \& Henderson, 2006b). This has implications for our experiments here. If the longer RTs allowed participants more experience with and, thus, more time to study the display then perhaps observers were becoming explicitly aware of the repetition. With explicit awareness, participants might be able to implement attentional guidance to help find the target. Although it will be left for future research to investigate this, a pattern seems to be emerging: keying participants into explicit recognition in contextual cueing seems to enable the use of attentional guidance.

Alternatively, it is possible that in complex search, with no bottom-up, exogenous cues, guidance may take time to develop. Thus when the display first appears, participants search it as if they were searching it from anew (see also Kunar, Flusberg \& Wolfe, submitted, Oliva et al., 2004 and Wolfe et al., 2000, for examples of this in a repeated search task). Here guidance systems would begin to be activated but would not yet be in full effect. If search was still in process when the guidance became available then that guidance could speed the search. Again it will be for future work to investigate this.

\section{Acknowledgements}

This research was supported by a grant from the National Institute of Mental Health to JMW. We wish to thank Kristin Michod for her assistance with data collection. 


\section{References}

Biederman I. Perceiving real-world scenes. Science 1972;177:77-80. [PubMed: 5041781]

Brainard DH. The Psychophysics Toolbox. Spatial Vision 1997;10:443-446. [PubMed: 9176954]

Brockmole JR, Castelhano MS, Henderson JM. Contextual Cueing in Naturalistic Scenes: Global and Local Contexts. Journal of Experimental Psychology: Learning, Memory, \& Cognition 2006;32:699_ 706.

Brockmole JR, Henderson JM. Recognition and attention guidance during contextual cueing in real-world scenes: Evidence from eye movements. The Quarterly Journal of Experimental Psychology 2006a; 59:1177-1187. [PubMed: 16769618]

Brockmole JR, Henderson JM. Using real-world scenes as contextual cues for search. Visual Cognition 2006b;13:99-108.

Chun MM. Contextual cueing of visual attention. Trends in Cognitive Science 2000;4:170-178.

Chun MM, Jiang Y. Contextual cueing: implicit learning and memory of visual context guides spatial attention. Cognitive Psychology 1998;36:28-71. [PubMed: 9679076]

Chun MM, Jiang Y. Top-down Attentional Guidance Based on Implicit Learning of Visual Covariation. Psychological Science 1999;10:360-365.

Chun MM, Jiang Y. Implicit, long-term spatial contextual memory. Journal of Experimental Psychology: Learning, Memory, \& Cognition 2003;29:224-234.

Egeth HE, Virzi RA, Garbart H. Searching for conjunctively defined targets. J. Exp. Psychol: Human Perception and Performance 1984;10:32-39.

Endo N, Takeda Y. Selective learning of spatial configuration and object identity in visual search. Perception \& Psychophysics 2004;66(2):293-302. [PubMed: 15129750]

Hoffmann J, Sebald A. Local contextual cuing in visual search. Experimental Psychology 2005;52(1): 31-38. [PubMed: 15779528]

Jiang Y, Chun MM. Selective Attention Modulates Implicit Learning. The Quarterly Journal of Experimental Psychology (A) 2001;54(4):1105-1124.

Jiang Y, Leung AW. Implicit learning of ignored visual context. Psychonomic Bulletin \& Review 2005;12 (1):100-106. [PubMed: 15948286]

Jiang Y, Leung A, Burks S. Source of individual differences in spatial context learning. Memory \& Cognition. (submitted)

Jiang Y, Song J-H, Rigas A. High-capacity spatial contextual memory. Psychonomic Bulletin \& Review 2005;12(3):524-529. [PubMed: 16235640]

Jiang Y, Wagner LC. What is learned in spatial contextual cuing - configuration or individual locations? Perception \& Psychophysics 2004;66(3):454-463. [PubMed: 15283070]

Kunar MA, Flusberg SJ, Wolfe JM. Contextual cueing by global features. Perception \& Psychophysics 2006;68:1204-1216. [PubMed: 17355043]

Kunar MA, Flusberg SJ, Wolfe JM. The role of memory and restricted context in repeated visual search. Perception \& Psychophysics. (submitted)

Kunar MA, Flusberg SJ, Horowitz TS, Wolfe JM. Does contextual cueing guide the deployment of attention? Journal of Experimental Psychology: Human Perception and Performance 2007;33:816828. [PubMed: 17683230]

Kunar MA, Michod KO, Wolfe JM. When we use context in contextual cueing: Evidence from multiple target locations. Journal of Vision 2005;5:412a.

Lleras A, Von Mühlenen A. Spatial context and top-down strategies in visual search. Spatial Vision 2004;17(4-5):465-482. [PubMed: 15559114]

Oliva A, Wolfe J, Arsenio H. Panoramic search: The interaction of memory and vision in search through a familiar scene. Journal of Experimental Psychology: Human Perception and Performance 2004;30:1132-1146. [PubMed: 15584820]

Olsen IR, Chun MM. Perceptual constraints on implicit learning of spatial context. Visual Cognition 2002;9(3):273-302.

Pelli DG. The VideoToolbox software for visual psychophysics: Transforming numbers into movies. Spatial Vision 1997;10:437-442. [PubMed: 9176953] 
Peterson MS, Kramer AF. Attentional guidance of the eyes by contextual information and abrupt onsets. Perception \& Psychophysics 2001;63:1239-1249. [PubMed: 11766947]

Treisman A, Gelade G. A feature-integration theory of attention. Cognitive Psychology 1980;12:97-136. [PubMed: 7351125]

Tseng Y, Li CR. Oculomotor correlates of context-guided learning in visual search. Perception \& Psychophysics 2004;66(8):1363-1378. [PubMed: 15813200]

Wolfe JM, Klempen N, Dahlen K. Postattentive vision. Journal of Experimental Psychology: Human Perception and Performance 2000;26:693-716. [PubMed: 10811170]

Wolfe JM, Oliva A, Horowitz TS, Butcher SJ, Bompas A. Segmentation of objects from backgrounds in visual search tasks. Vision Research 2002;42:2985-3004. [PubMed: 12480070] 
a) Wall Condition
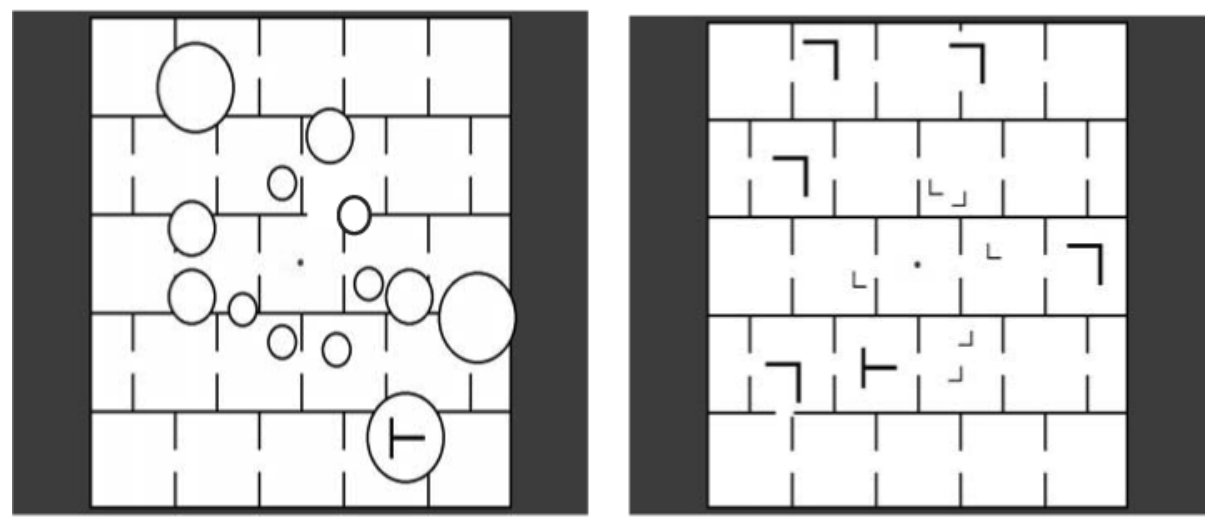

b) Standard Condition

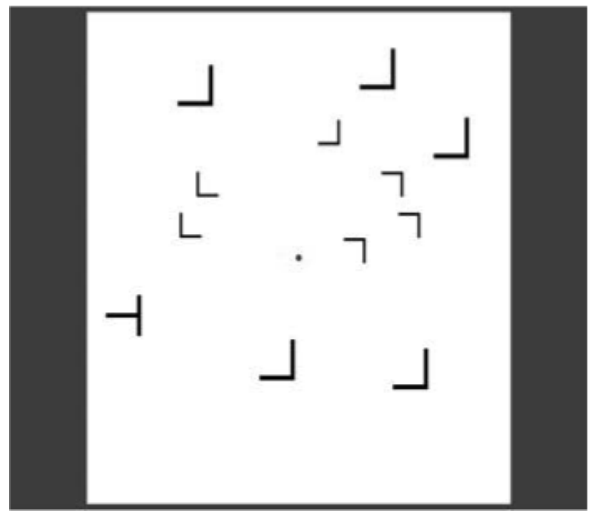

Figure 1.

Example displays for Experiment 1. Figure 1a shows an example from the Wall condition (set sizes 1 and 12), whereas Figure 1b shows an example for the standard contextual cueing condition (set size 12). 


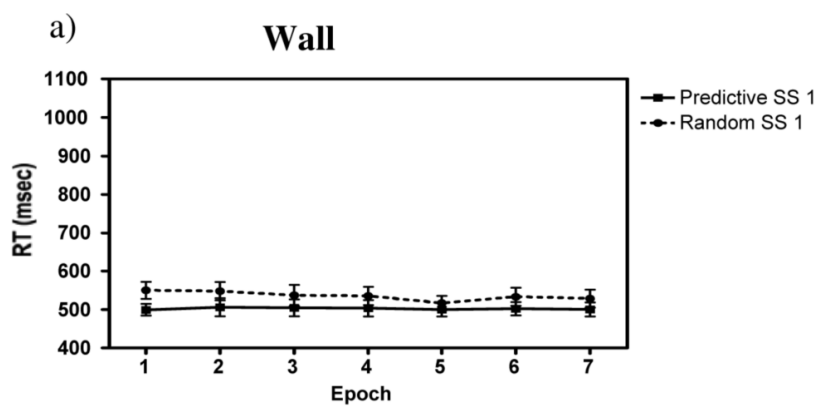

b)

Wall
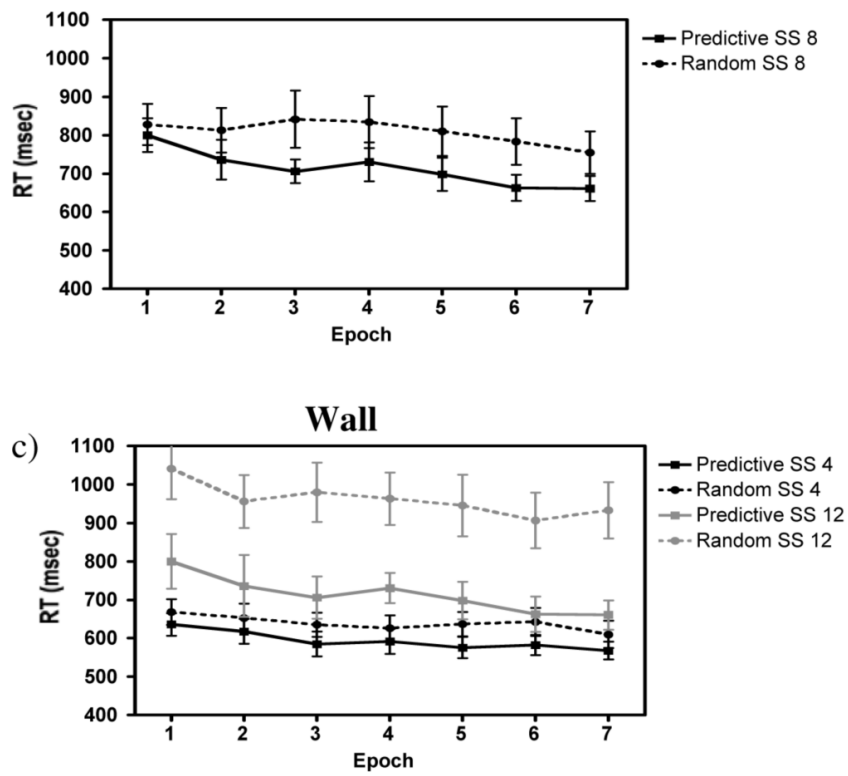

d)

Standard

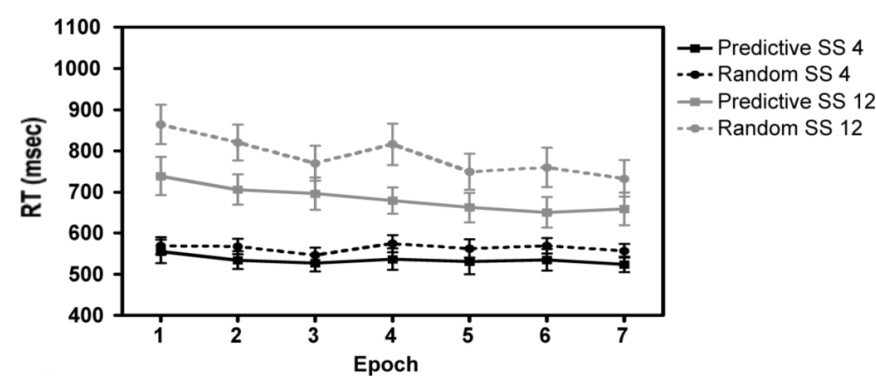

Figure 2.

Mean correct RTs (msec) across epoch for Experiment 1 (a) set size 1, (b) set size 8, (c) set size 4 and 12 of the Wall condition and (d) set size 4 and 12 of the standard contextual cueing condition. 
a)

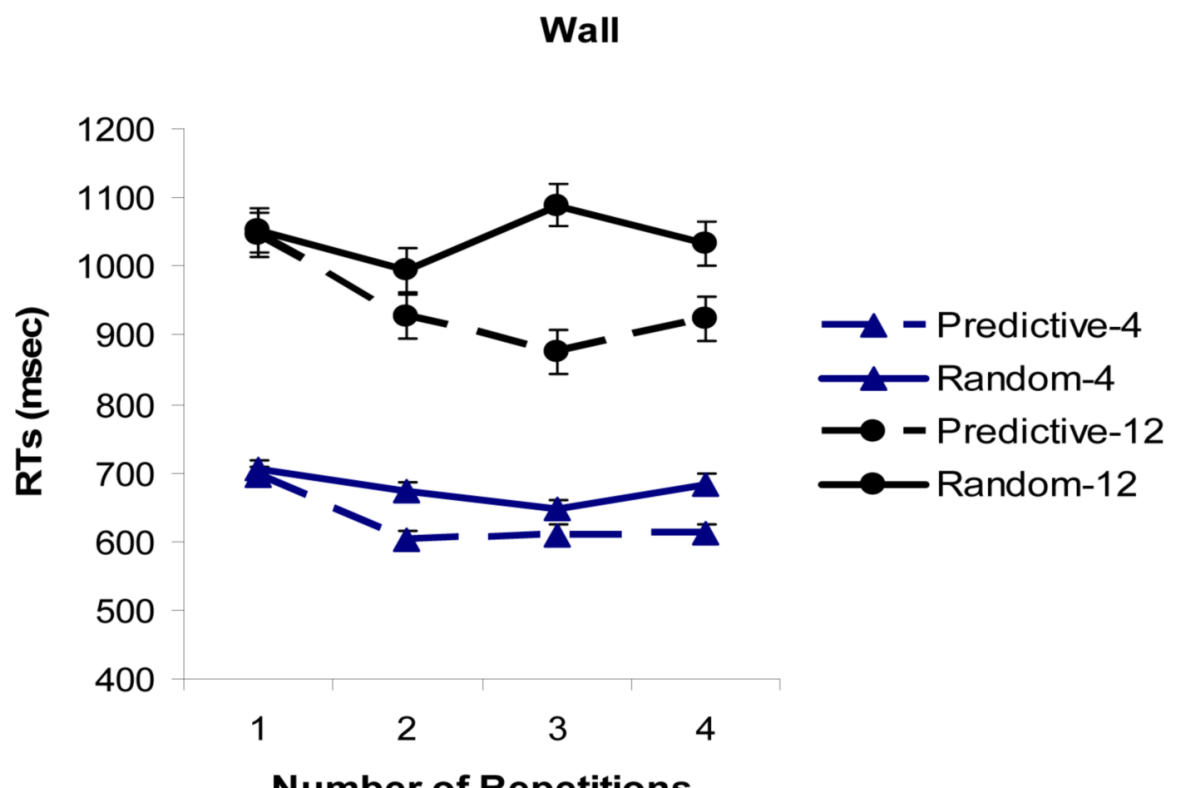

b)

\section{Standard}

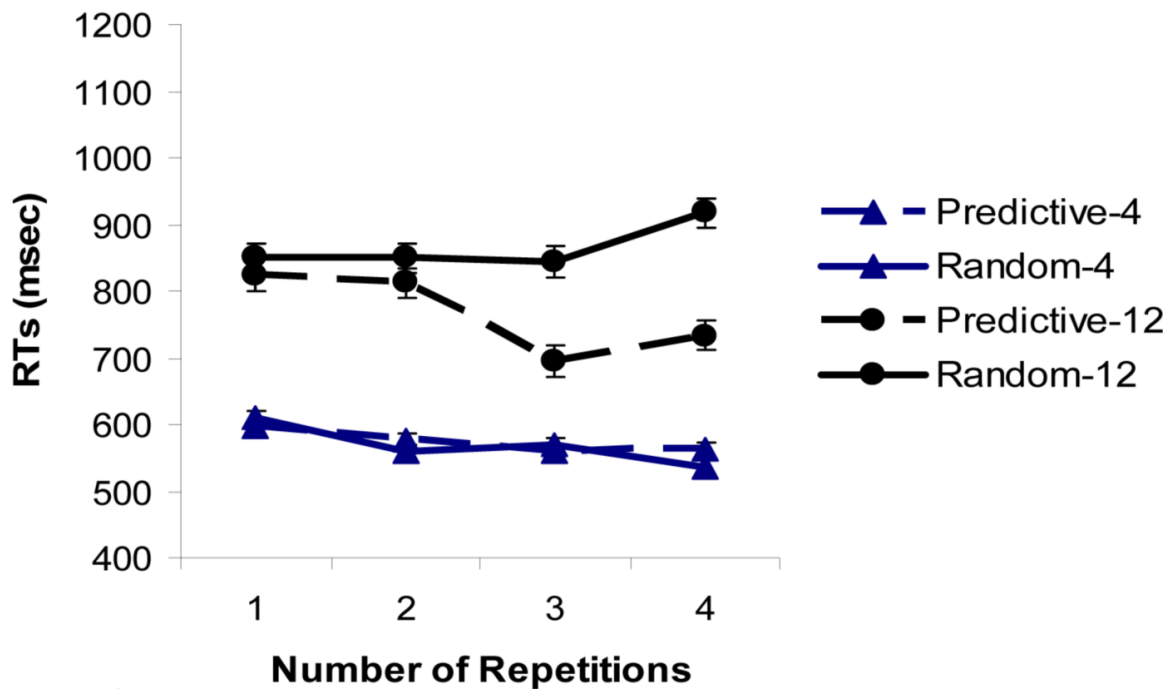

Figure 3.

Mean correct RTs (msec) across the first four repetitions of the (a) Wall condition and (b) Standard contextual cueing condition for both set sizes 4 and 12 . 
a)

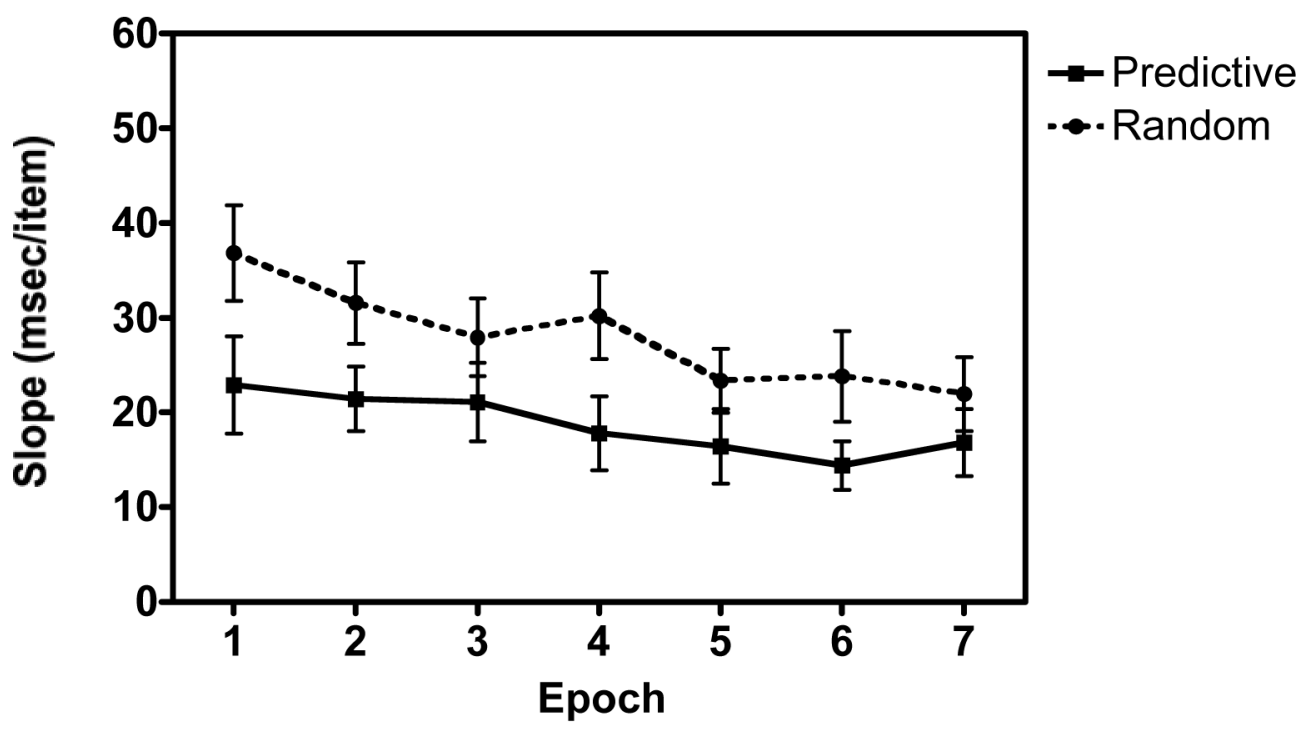

b)

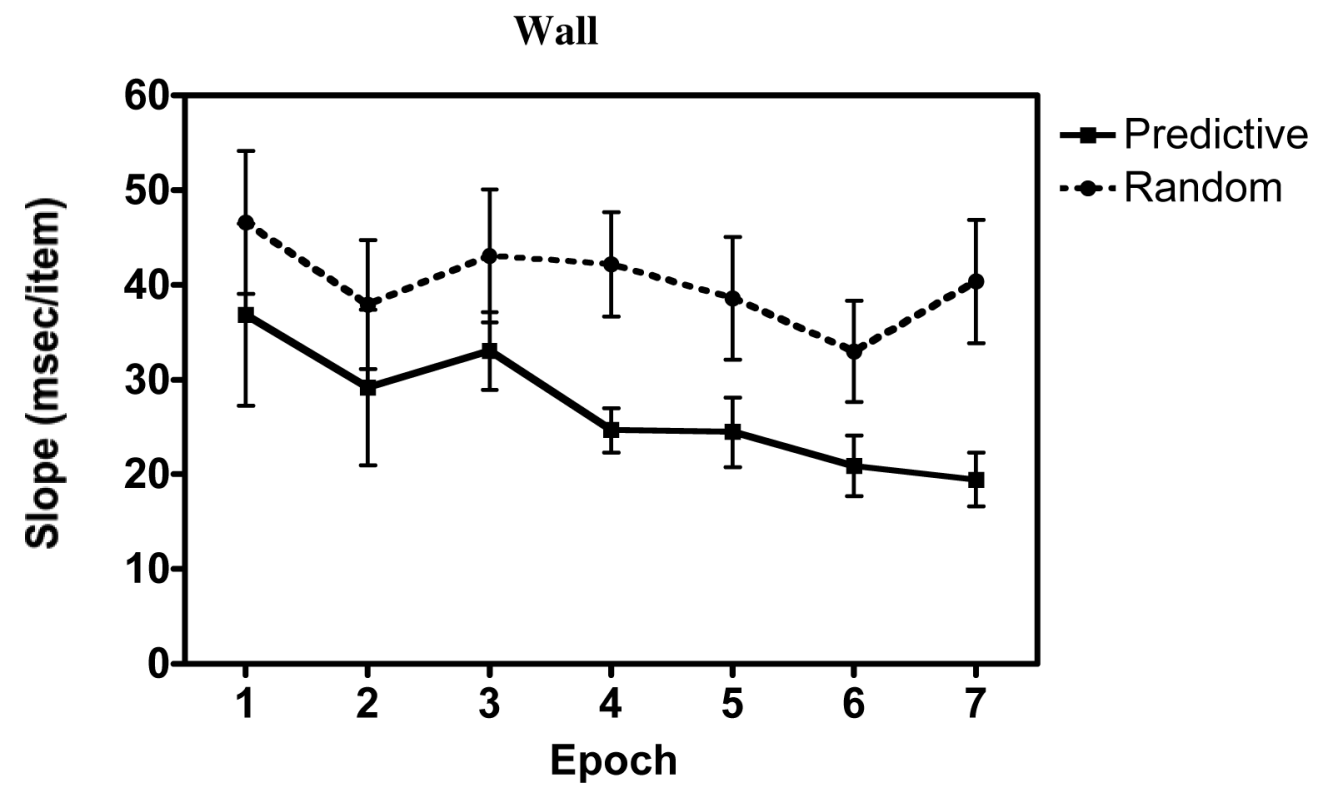

Figure 4.

Search slopes (msec/item) for each condition over epoch in Experiment 1. Figure 4a shows search slopes in the Standard contextual cueing task, whereas Figure $4 \mathrm{~b}$ shows those from the Wall condition. 


\section{Standard Contextual Cueing}

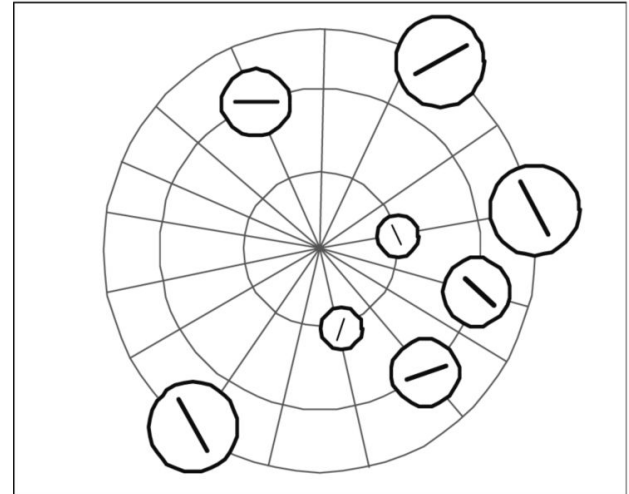

\section{SOA Onset}
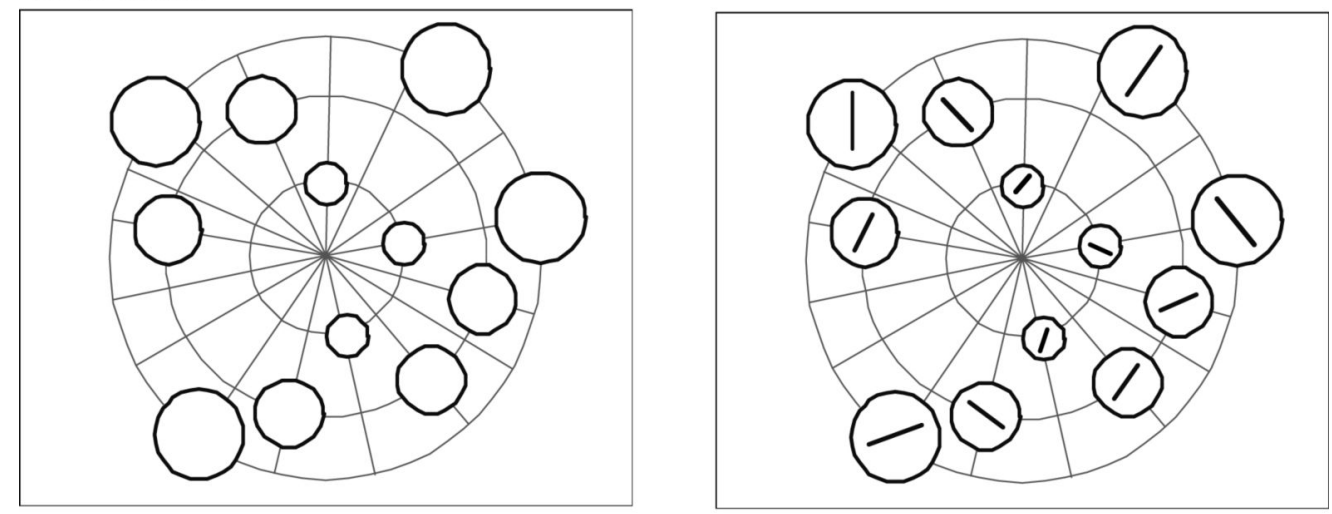

\section{SOA Offset}
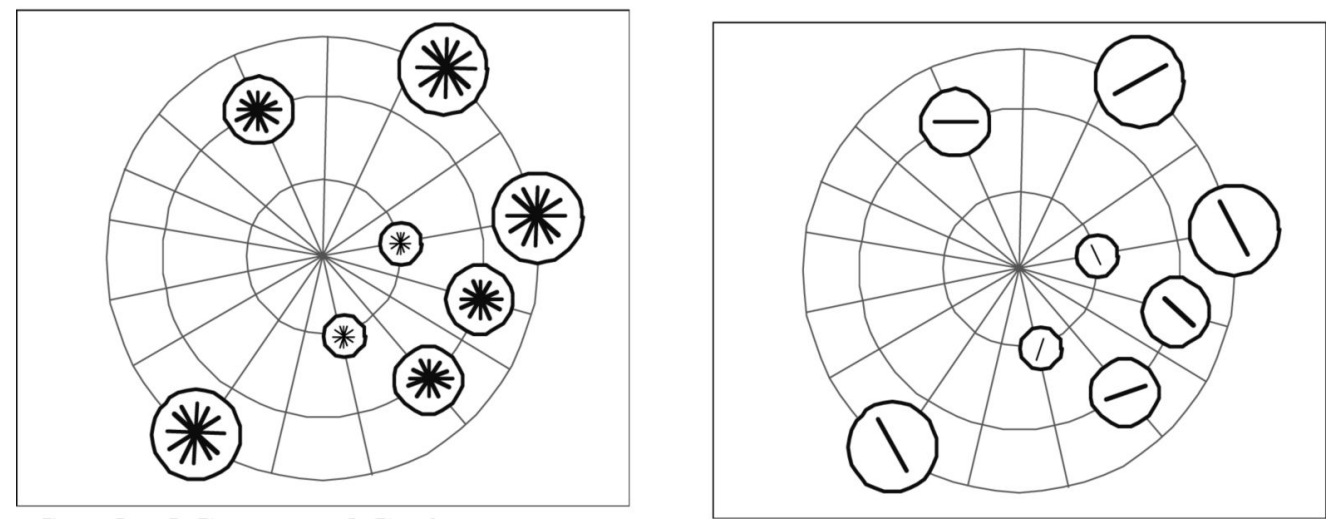

Figure 5.

Example displays for each condition in Experiment 2. 
a)

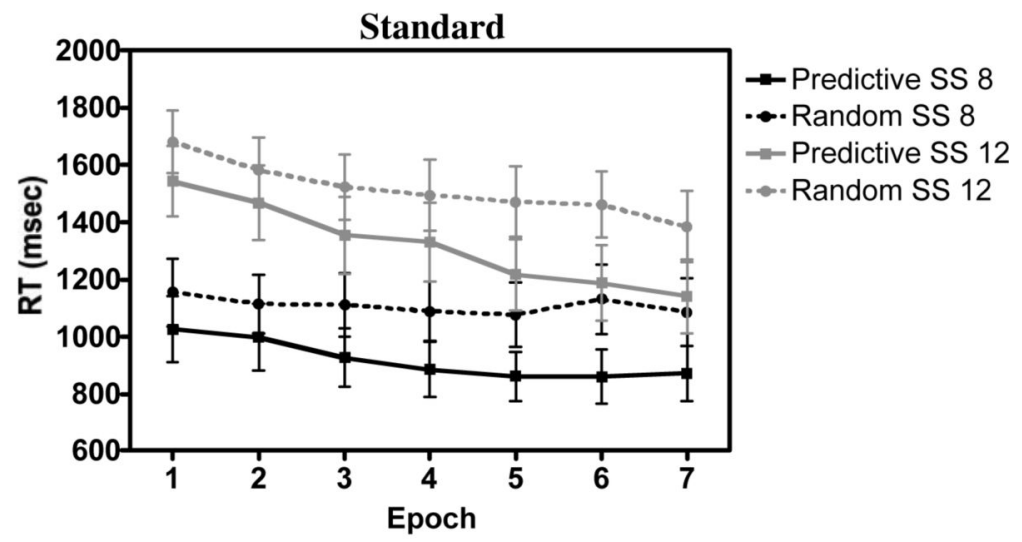

b)

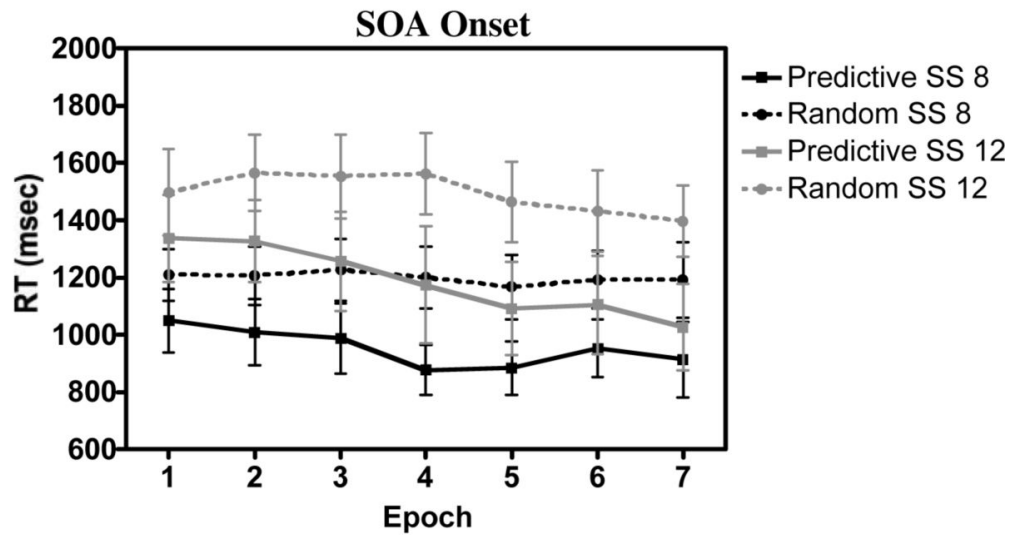

c)

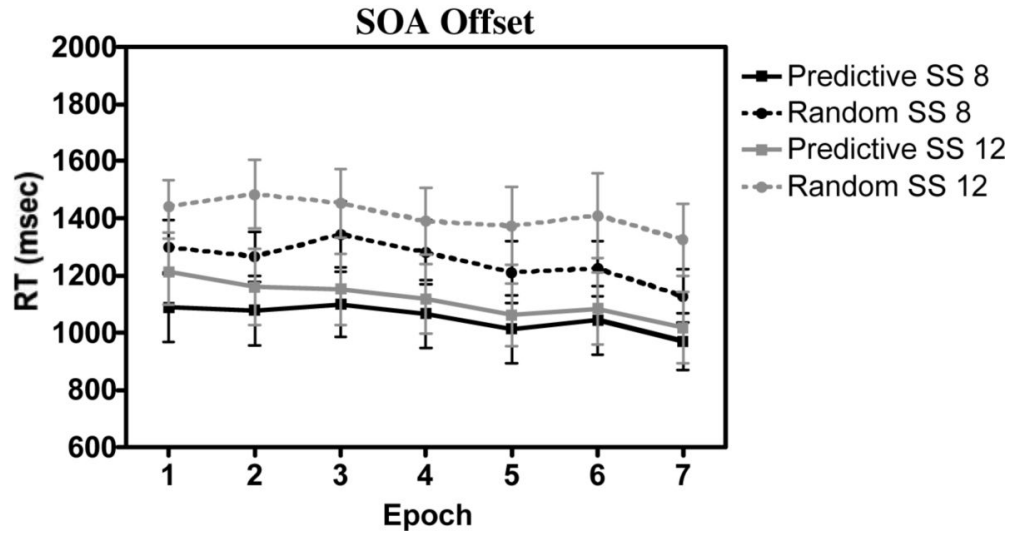

Figure 6.

Mean correct RTs (msec) across epoch for Experiment 2 for (a) the Standard contextual cueing, (b) SOA Onset and (c) SOA Offset conditions. 
a)

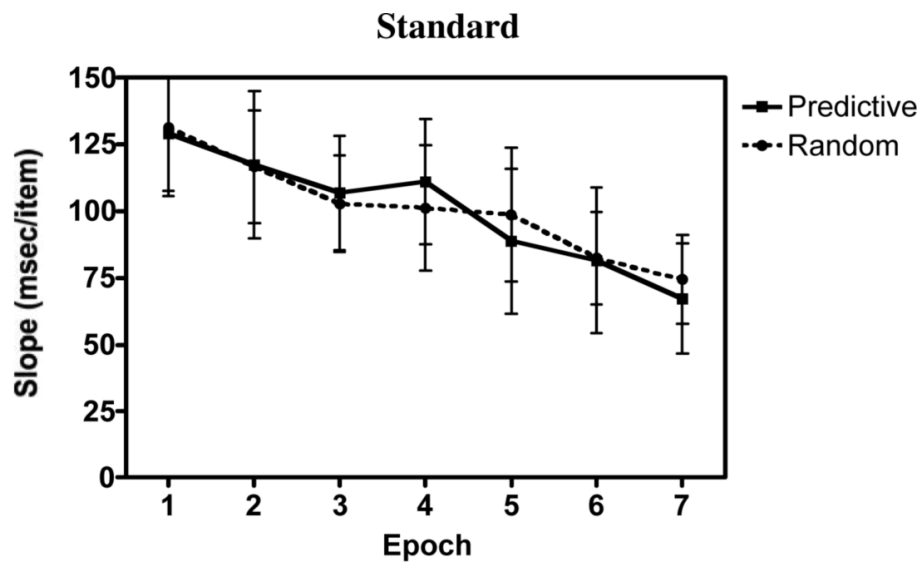

b)

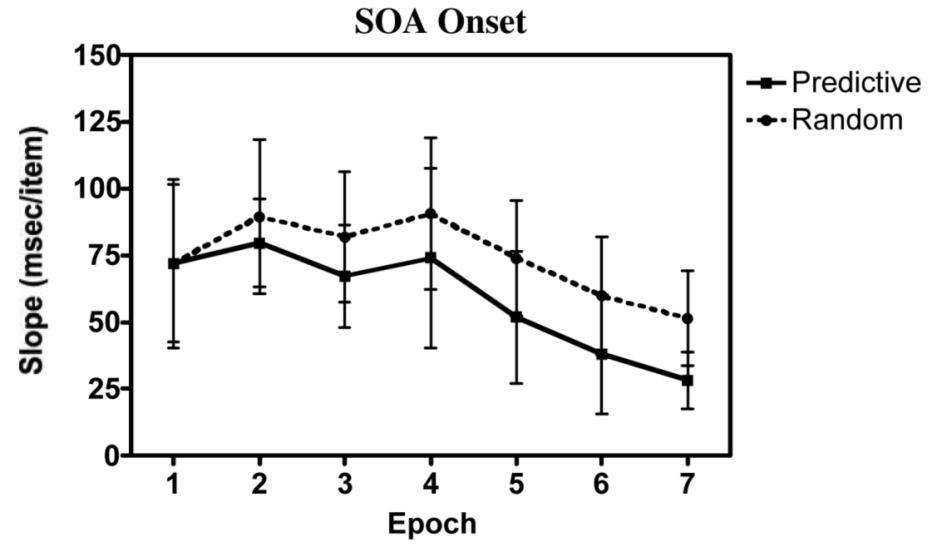

c)

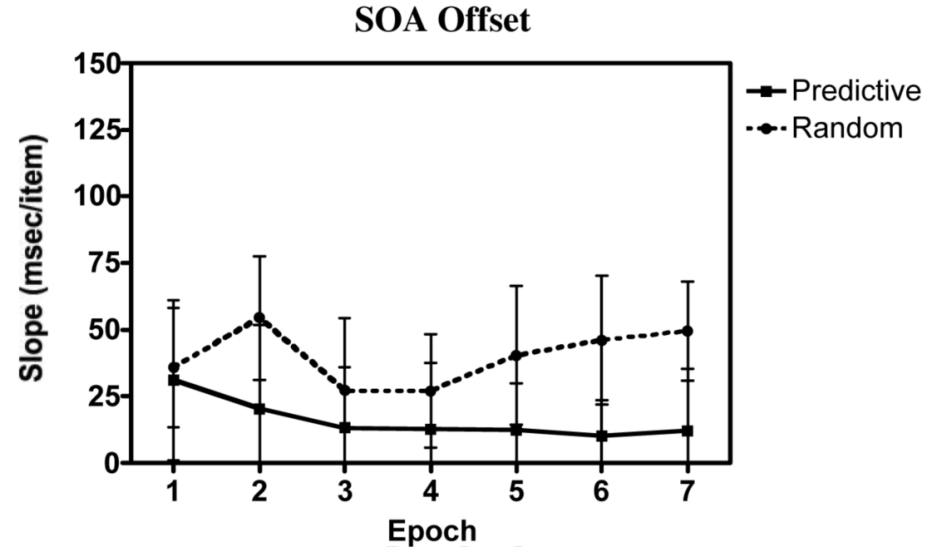

Figure 7.

Search slopes (msec/item) for each condition over epoch in Experiment 2. Figure 8a shows search slopes in the Standard contextual cueing task, Figure $8 \mathrm{~b}$ shows those the SOA Onset condition, whereas Figure 8c shows those for SOA Offset. 


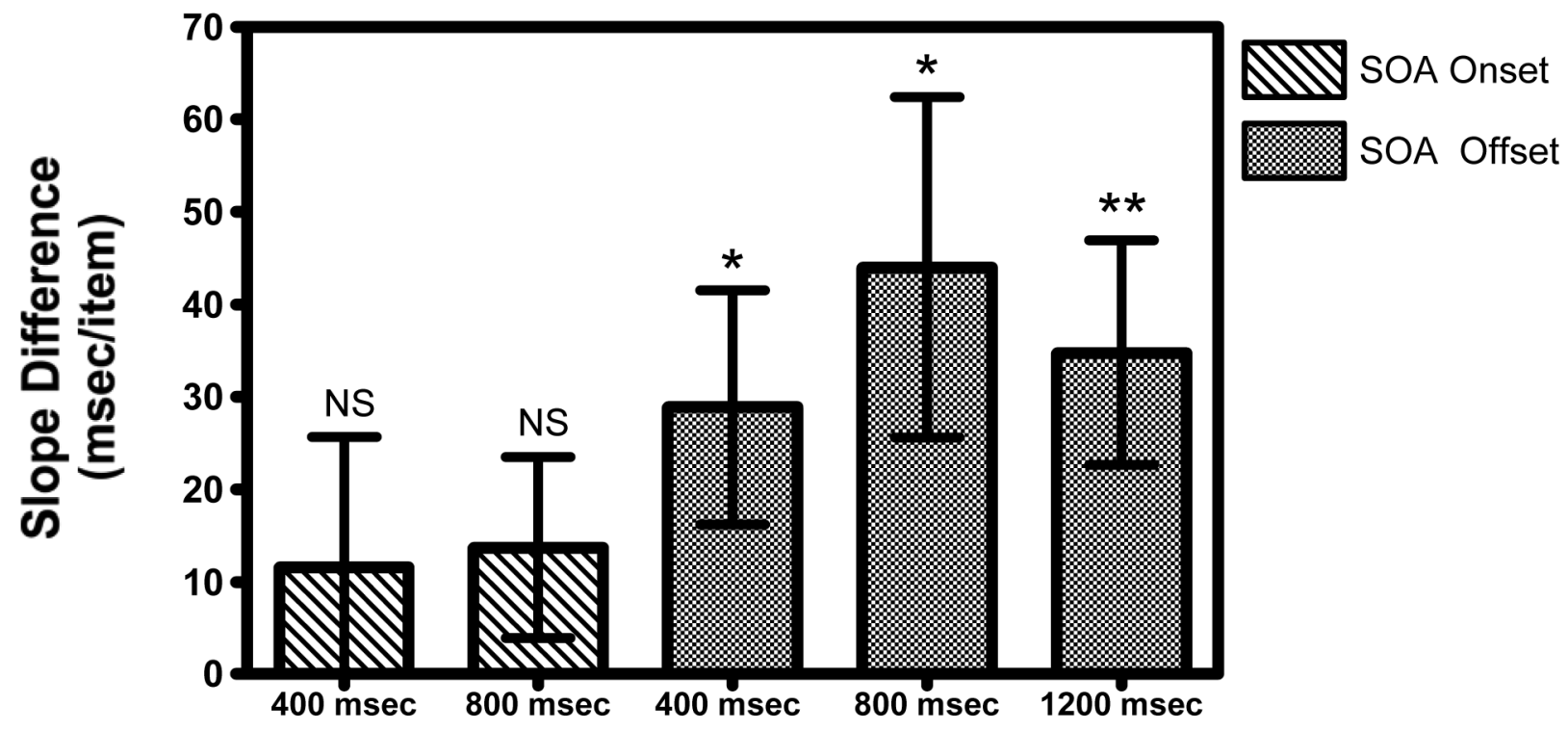

Figure 8.

The difference in search slopes (random minus predictive), across the last 3 epochs, for replications of SOA Onset with SOAs of 400 and 800 msecs and SOA Offset with SOAs of 400, 800 and $1200 \mathrm{msec}$. 


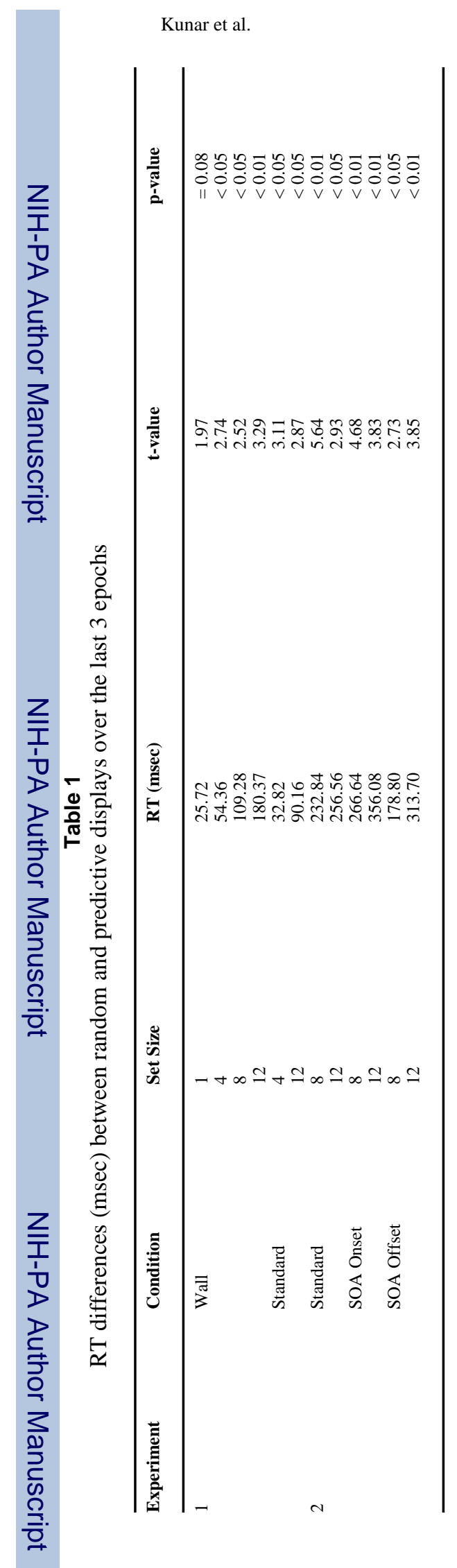

Vis cogn. Author manuscript; available in PMC 2008 October 8. 


\section{Table 2}

The difference in search slopes (random minus predictive) for both the Wall and Standard contextual cueing conditions, in Experiment 1, across the last 3 epochs and at Epochs 1 and 7. Standard error values are shown in parentheses.

\begin{tabular}{cccc}
\hline Condition & $\begin{array}{c}\text { Slope difference across the last 3 } \\
\text { epochs (msec/item) }\end{array}$ & $\begin{array}{c}\text { Slope difference at Epoch 1 } \\
(\mathbf{m s e c} / \text { item })\end{array}$ & $\begin{array}{c}\text { Slope difference at Epoch 7 } \\
(\mathbf{m s e c} / \text { item) }\end{array}$ \\
\hline Wall & $15.6(4.8)$ & $9.7(7.7)$ & $21.0(5.2)$ \\
Standard & $7.2(3.7)$ & $14.0(5.5)$ & $5.1(4.3)$ \\
\hline
\end{tabular}




\section{Table 3}

The difference in search slopes across the last 3 epochs (random minus predictive) for all conditions in Experiment 2. Standard error values are shown in parentheses.

\begin{tabular}{cc}
\hline Condition & Slope difference across the last 3 epochs (msec/item) \\
\hline Standard & $5.9(22.3)$ \\
SOA Onset & $22.4(20.5)$ \\
SOA Offset & $33.7(11.6)$ \\
\hline
\end{tabular}

